DOI: $10.14451 / 1.178 .123$

\title{
КОНЦЕПЦИИ ЛОГИСТИКИ В ТРАНСПОРТНОМ АСПЕКТЕ
}

\author{
(c) 2019 Сосунова Лильяна Алексеевна \\ доктор экономических наук, профессор \\ Самарский государственный экономический университет, Россия, Самара \\ E-mail: kafedra-kl@yandex.ru
}

Обосновывается положение, согласно которому логистические концепции могут быть в полной мере осуществлены при развитом транспортном обеспечении. Первостепенное положение приобретает транспортное содержание логистических концепций.

Ключевые слова: логистические концепции, транспорт, цепи поставок, снабжение и сбыт, поставщики и потребители, «точно в срок», запасы, заказы.

K настоящему времени логистика - как управление потоковыми процессами в экономике - претерпела весьма существенную трансформацию в направлении усиления транспортной составляющей.

Материальные потоки не могут осуществляться без транспорта: потоковым процессам товародвижения необходимы «колеса» в виде транспорта различного вида и назначения.

Концепции логистики или логистические концепции представляют собой совокупность специальных правил и методов организации и управления товародвижением, основанных на понимании производственно-коммерческой деятельности как потокового процесса. Указанные правила и методы, обусловленные концепциями логистики, служат инструментом формирования особых управляющих воздействий на потоковые процессы движения материальных и товарных потоков в предпринимательской деятельности. С помощью концепций логистики реализуется специфика в управлении производственно-коммерческой деятельностью в пред- принимательских структурах.

По своей сущности концепции логистики представляют собой концептуальные системы в форме алгоритмов производственно-коммерческой деятельности предпринимательства.

В отечественной экономике логистика использовалась для управления процессами товародвижения, что предопределило её коммерческую направленность. Именно поэтому в логистических концепциях доминирующее положение заняла коммерческая направленность. В связи с этим представляется необходимым кратко рассмотреть основное содержание логистических концепций (табл. 1).

Как следует из таблицы, каждая логистическая концепция может быть в полной мере реализована в производственно-коммерческой деятельности только при наличии развитого транспортного обеспечения. При этом логистика создает необходимые условия для транспортной доступности субъектов (предприятий) и объектов (продукции, товаров).

Следовательно, логистические концепции

Таблица 1. Основные логистические концепции

\begin{tabular}{|c|l|l|l|}
\hline $\begin{array}{c}\text { №№ } \\
\text { пп }\end{array}$ & \multicolumn{1}{|c|}{$\begin{array}{c}\text { Наименование } \\
\text { международное }\end{array}$} & \multicolumn{1}{|c|}{ Обозн. } & \multicolumn{1}{|c|}{$\begin{array}{c}\text { Наименование } \\
\text { общепринятое }\end{array}$} \\
\hline 1 & Supply Chain Management & (SCM) & «Управление цепями поставок». \\
\hline 2 & $\begin{array}{l}\text { Materials / manufacturing } \\
\text { requirements / resource planning }\end{array}$ & (MRP) & $\begin{array}{l}\text { «Система планирования потребностей в материалах / } \\
\text { планирование потребностей производства в ресурсах». }\end{array}$ \\
\hline 3 & $\begin{array}{l}\text { Distribution requirements } \\
\text { planning }\end{array}$ & DRP & $\begin{array}{l}\text { Подсистема/модуль - планирование дистрибьюции } \\
\text { готовой продукции. }\end{array}$ \\
\hline 4 & $\begin{array}{l}\text { Customer sinchronized resource } \\
\text { planning }\end{array}$ & CSRP & $\begin{array}{l}\text { Планирование ресурсов, синхронизированное с потре- } \\
\text { бителем. }\end{array}$ \\
\hline 5 & Just-in-Time & JIT & «Точно в срок». \\
\hline 6 & Effective Customer Response & ECR & «Эффективная реакция на запросы потребителей». \\
\hline 7 & Vendor Managed Inventory & VMI & $\begin{array}{l}\text { Логистическая система управления спросом и пополне- } \\
\text { ния запасов «Управление запасами поставщиком». }\end{array}$ \\
\hline
\end{tabular}


сохраняют свое практическое назначение, но с преобладанием транспортного содержания. В связи с этим необходимо рассмотреть концепции логистики в транспортном аспекте.

I. Из логистических концепций первой считается концепция SCM - «Управление цепями поставок». Формирование цепи поставок в значительной мере представляет собой планирование маршрута следования поставляемой продукции от начального пункта к конечному звену - предприятиям розничной торговли - прямо или через промежуточные звенья. При этом следует иметь в виду, что поставщики и потребители могут быть на нескольких уровнях, что предопределяет многовариантность траектории цепи поставки. А поэтому цепи поставок описываются с помощью модели транспортной задачи линейного программирования.

Модель транспортной задачи линейного программирования имеет следующее содержание (в терминах цепи поставок):

Продукция, сосредоточенная в $m$ поставщиках (пунктах отправления) в количествах $a_{1}, a_{2}$, $\ldots, a_{\mathrm{m}}$, необходимо доставить в каждый из $n$ потребителей (пунктов назначения) в количествах $b_{1}, b_{2}, \ldots, b_{\mathrm{n}}$. Стоимость поставок из $i$-го поставщика $j$-му потребителю равна $c_{i j}$. Следует определить оптимальный план поставок, т.е. найти $x_{i j}$ для этого оптимального плана.

В общем виде модель линейного программирования состоит из целевой функции и ограничений (условий). Для транспортной задачи целевая функция имеет следующий вид:

$$
L=\sum_{i=1}^{m} \sum_{j=1}^{n} C_{i j} X_{i j}=\min
$$

Целевая функция предусматривает минимизацию поставки всей продукции из пункта і в пункт j.

Ограничения:

$$
\sum_{i=1}^{m} x_{i j}=b_{i} \quad \sum_{j=1}^{n} x_{i j}=a_{i} \quad x_{i j} \geq 0
$$

Ограничения показывают, что вся имеющаяся продукция, сосредоточенная во всех $i$-х пунктах отправления, должны быть полностью доставлены в требуемых количествах в пункты потребления $j$.

Представленная модель в значительной мере носит иллюстративный характер и как ма- тематическая модель способствует углубленному пониманию сущности цепей поставок.

В цепи поставок разрозненные предприятия объединяются в систему с помощью виртуального оператора. В цепи поставок отсутствует такой оператор в виде определенной организационной структуры, стоящей над всеми звеньями цепи. Однако в неформальном виде такой оператор присутствует - таким оператором является персонал логистического менеджмента каждого звена. [2, с. 19-24].

Необходимым условием функционирования такого неформального оператора является использование персоналом в максимальной степени логистических концепций.

Такие концепции присущи всем участникам цепи поставок как производственным, так и торговым предприятия. Но при этом ключевую роль в достижении совместного и согласованного действия играет концепция SCM - в этой связи достаточно отметить её модуль «Управление взаимоотношениями с потребителями», поскольку каждое последующее звено цепи является потребителем продукции или услуг. Согласно теории логистики, концепция SCM есть «интегрированная логистика», вследствие чего данная концепция определяет единство действий всех участников цепи поставок.

II. Центральное место в производственно-коммерческой деятельности занимает концепция MRP - «Система планирования потребностей в материалах / планирование потребностей производства в ресурсах». Согласно данной концепции, потребность в материальных ресурсах для нужд материально-технического снабжения должна определяться на всю производственную программу строго по нормам расхода методом «прямого счета». В рассматриваемом аспекте - одновременно с определением потребности в материальных ресурсах - следует определять потребность в транспорте с полной его инфраструктурой, включая график доставки ресурсов на предприятие. Кроме того, данная концепция должна регулировать внутрипроизводственный транспорт - доставка материалов и полуфабрикатов непосредственно на рабочие места.

III. Концепция/модуль DRP используется в системах распределения готовой продукции. Основной инструмент логистического менеджмента в системе DRP представляет собой график, который координирует весь процесс поста- 
вок и пополнения запаса готовой продукции в дистрибутивной сети. Данная концепция относится к прогрессивным методам сбыта.

Система DRP обладает следующими маркетинговыми достоинствами:

- улучшение уровня обслуживания за счет сокращения времени доставки готовой продукции и удовлетворения требований потребителей;

- улучшение продвижения новых товаров на рынок;

- способность продвигать готовую продукцию с низким уровнем запасов;

- лучшая координация управления товарными запасами готовой продукции с другими функциями фирмы-предприятия;

- возможность удовлетворять требования потребителей за счет сервиса, связанного с координацией управления товарными запасами готовой продукции.

Логистические преимущества системы DRP включают:

- сокращение логистических издержек, связанных с управлением запасами готовой продукции путем координации поставок;

- сокращение запасов за счет более точного определения размера, места и сроков поставок;

- снижение потребности в складских площадях за счет сокращения запасов;

- сокращение транспортной составляющей логистических издержек за счет организации более эффективной обратной связи с потребителями;

- координация между производственными операциями и операциями по распределению продукции.

Среди логистических достоинств обращают на себя внимание транспортные составляющие, а именно: координация поставок, что достигается за счет диспетчеризации перевозок, а также сокращение транспортной составляющей издержек. При этом и другие достоинства также в той или иной мере обусловлены транспортным фактором.

IV. Концепция управления производственными ресурсами - CSRP - предусматривает планирование ресурсов, синхронизированное с потребителем.

Планируются не только основные, но и вспомогательные ресурсы. Эти ресурсы используются для оперативной работы с потребителями, включая послепродажное обслуживание. Рас- сматриваемая концепция может быть охарактеризована следующим образом:

- интегрированная система поддержания жизненного цикла товара;

- является инструментом оперативного изменения производственного графика в зависимости от изменения заказов потребителями;

- инструмент «тонкой» настройки производственного графика, осуществляемое во взаимодействии с потребителями;

- способность производителя удовлетворять особые и уникальные требования потребителя.

Выполнение системы CSRP - сопряжено с увеличением затрат, обусловленных переналадкой оборудования, срочными закупками необходимых материальных ресурсов, а также потерями от возможного несвоевременного выполнения уже размещенных заказов. Однако определенная доля таких затрат может быть покрыта транспортной составляющей за счет быстрейшего реагирования на запросы потребителя и скорейшей доставки необходимых ресурсов. А это означает, что для осуществления на практике данной концепции транспорт должен находиться в постоянной готовности.

V. Концепция JIT - Just-in-Time - «Точно в срок» предусматривает доставку необходимых материальных ресурсов или товаров в необходимое время в нужное место,

или

«Точно в срок» это поставка материальных ресурсов, непосредственно под их запуск в производство или поставки товаров в торговую сеть непосредственно на места в торговых залах,

или

«Точно в срок» - синхронизация процесса поставок материальных ресурсов с календарным графиком производства.

Логистическая концепция «точно в срок» обеспечивает работу предприятий-товаропроизводителей практически без производственных запасов, а работу предприятий розничной сети - без складских товарных запасов. Такая система поставок достигается при закупках материальных ресурсов и товаров у относительно небольшого числа надежных поставщиков и перевозчиков. Для концепции «точно в срок» требуются высокое качество готовой продукции и логистического обслуживания, поскольку исключается приемка поставляемой продукции потребителем. 
Материальные ресурсы, поставляемые по системе “точно в срок”, приходят на предприятие в максимальной технологической готовности с полной гарантией поставщика качества и количества поставляемой продукции (рис. 1). Из приведенной схемы видно, что поставки по системе “точно в срок” представляют собой синхронизацию графика производства и графика поставок - вплоть до полного совпадения временных параметров, т.е.

$\mathrm{Ti}-\mathrm{ti}=\Delta$ при этом $\Delta \approx 0$.

Преимущества системы «точно в срок»:

- низкий уровень запасов материальных ресурсов, незавершенного производства, готовой продукции;

- сокращение производственных площадей;

- повышение качества продукции;

- сокращение сроков производства;

- повышение гибкости производства при изменении ассортимента производимой продукции;

- надежный и стабильный поток производства;

- высокая производительность и эффективность использования оборудования;

- хорошие отношения с поставщиками;

- сокращение непроизводственных (вспомогательных) работ, например, складирование и внутрипроизводственное перемещение материальных ресурсов.

При поставках «точно в срок» основная масса запасов сосредоточена в специальных оптово-торговых предприятиях - Логистических Центрах или Распределительных Центрах. Такие Центры для надежности поставок имеют собственный или арендуемый транспорт, а также постоянные логоворные отношения с транспортными организациями.

VI. Концепция Effective Customer Response ECR - «Эффективная реакция на запросы потребителей».

Концепция ECR есть применение концепции «Точно в срок» в дистрибьюции готовой продукции, в частности, потребительских товаров. Является развитием метода «Быстрого реагирования» (QR) на запросы потребителей предусматривает использование товаропроизводителями и розничными магазинами компьютеризированных систем для автоматической обработки заказов. Для данной концепции, как правило, используется малотоннажный транспорт высокой мобильности.

VII. Логистическая система управления спросом и пополнения запасов - Vendor Managed Inventory - VMI - «Управление запасами поставщиком».

Управление запасами у потребителя с помощью обмена информацией с поставщиком. Эта информация содержит сведения о фактическом спросе или продажах продукции потребителем-продавцом и имеющихся запасах у поставщика-продавца на данный момент. На основе этой информации поставщик берет на себя ответственность за пополнение запасов потребителя.

В системе VMI отпадает надобность в заказах на поставку продукции: потребителям сообщается информация о минимальных и максимальных пределах запасов, которые им разрешается иметь, а поставщик несет ответственность за поддержание необходимого объема запасов у

\section{Моменты запуска материалов в производство}

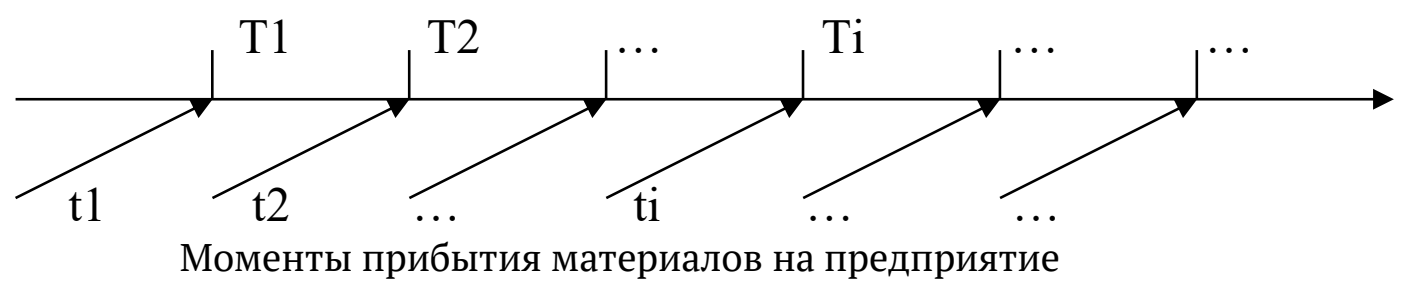

Обозначения на схеме:

$\mathrm{Ti}$ - моменты времени запуска материалов в производство;

$\mathrm{ti}$ - моменты прибытия материалов на предприятие.

Puc. 1. Схема организации поставок “точно в срок” 
потребителя.

В системе VMI соглашения строятся на тесном сотрудничестве между потребителем и поставщиком: по существу имеет место «совместное управление запасами». Система VMI иллюстрируется схемой (рис. 2).

Транспортное содержание основных логистических концепций приведено в табл. 2.

Рассмотренные логистические концепции носят типовой характер и конкретизируются предпринимателем в зависимости от условий своей производственно-коммерческой деятельности.

Итак, роль транспорта в логистике:
1) сопряжение - интерфейс - звеньев в цепи поставок;

2) надежность снабжения и сбыта;

3) своевременное получение дохода от сбыта - отгрузки продукции;

4) оперативное маневрирование материальными ресурсами;

5) обеспечение работы без запасов за счет концепций JiT и VMI;

6) сокращение издержек в производственно-коммерческой деятельности;

7) мобильность, динамизм и ускорение оборачиваемости ресурсов;

8) достижение всеобщего оптимума в це-

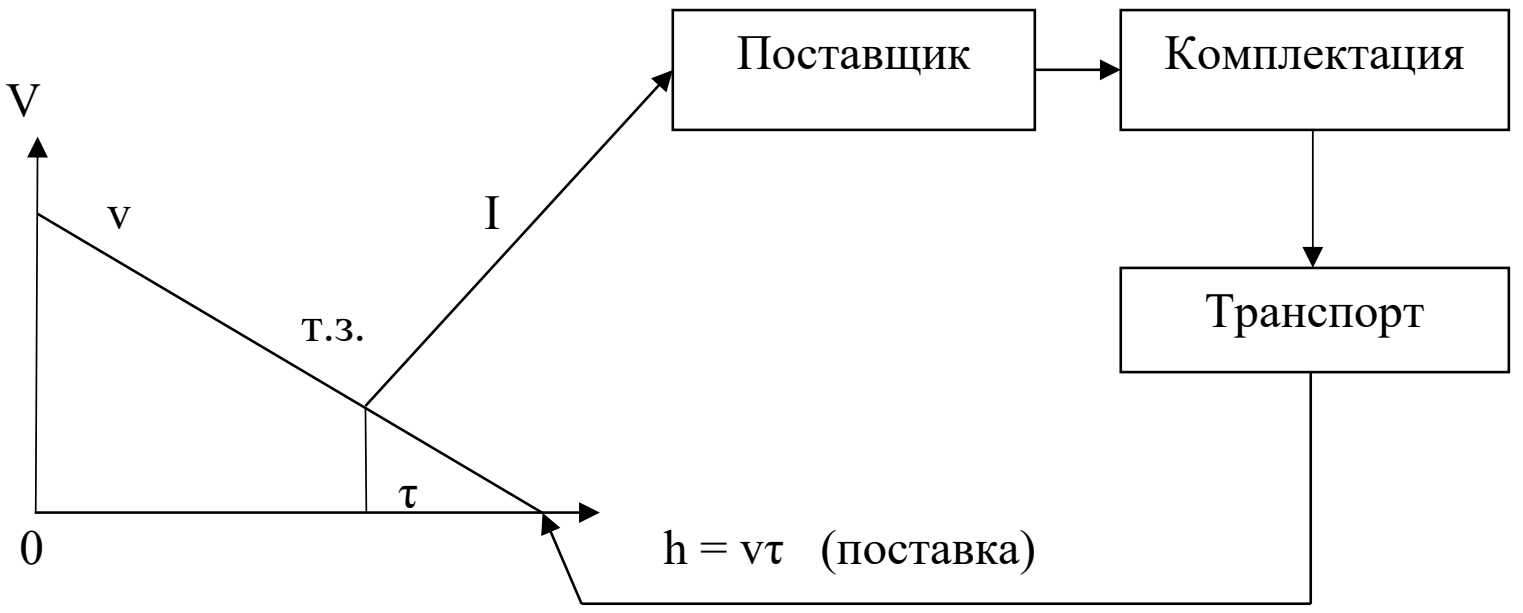

Обозначения:

$\mathrm{V}$ - расходуемый запас;

v - интенсивность расхода запаса;

т.з. - «точка заказа»;

$\tau$ - время выполнения заказа;

I - информация о достижении «точки заказа» и о величине заказа;

$\mathrm{h}$ - величина партии поставки.

Puc. 2. Схема концепции VMI

Таблица 2. Транспортное содержание логистических концепций

\begin{tabular}{|c|c|l|}
\hline $\begin{array}{c}\text { №№ } \\
\text { пп }\end{array}$ & $\begin{array}{c}\text { Обозначе- } \\
\text { ния }\end{array}$ & \multicolumn{1}{|c|}{ Транспортное содержание } \\
\hline 1 & SCM & Оптимизация маршрутов транспортировки в цепях поставок \\
\hline 2 & MRP & Доставка исходных материальных ресурсов \\
\hline 3 & DRP & Отгрузка продукции потребителям \\
\hline 4 & CSRP & Оперативная реакция перевозчика \\
\hline 5 & JiT & Доставка продукции под запуск в производство или товаров в торговый зал на выкладку \\
\hline 6 & ECR & Мобильная доставка по заявкам потребителей \\
\hline 7 & VMI & Пополнение поставщиком запаса по «точке заказа» потребителя \\
\hline
\end{tabular}


пях поставок в форме взаимной коммерческой выгоды - оптимум по Парето;

9) расширение функций транспорта как отрасли материального производства за счет интеграции логистических концепций.

Таким образом, применение логистических концепций переводят на качественно более высокий уровень осуществления предпринимательской деятельности, как в сфере произ- водства, так и в сфере обращения. Указанные концепции, с одной стороны, регламентируют управленческие процедуры, а с другой стороны, реализуют креативный потенциал управленческого персонала предпринимательских структур. При этом достигается единство коммерческой и транспортной составляющих каждой концепции.

\section{Библиографический список}

1. Плоткин Б.К. Экономика: предпринимательство, логистика и цепи поставок - СПб.: Изд-во Инфо-да, 2015.- $131 \mathrm{c}$.

2. С.В. Носков, Н.П. Карпова, И.А. Тойменцева, Е.В. Швецова, Факторы эффективности железнодорожных перевозок, Экономика и предпринимательство, № 8-1 (85-1). С.569-573, 2017, импакт-фактор 0,345.

3. T.Е. Евтодиева, Современные концепции логистики: содержительный аспект, European Social Science Journal, № 12 (1). С. 72-80, 2016, импакт-фактор 0,102

4. Д.В. Чернова, Н.А. Крючкова, А.А. Туренко, Тенденции развития логистической инфраструктуры автомобильного и железнодорожного транспорта Вестник Самарского государственного экономического университета, № 12 (122). С. 108-113, 2014, импакт-фактор 0,652 